\title{
Transcriptional and epigenetic regulation in human islets
}

\author{
Nuria C. Bramswig • Klaus H. Kaestner
}

Received: 16 September 2013 / Accepted: 6 December 2013 /Published online: 21 December 2013

(C) Springer-Verlag Berlin Heidelberg 2013

\begin{abstract}
Gene regulation in human pancreatic endocrine cells is a complex process, governed by genetic and environmental factors and crosstalk between the various endocrine cell types, and between endocrine cells and the metabolic state. Recent advances in gene expression profiling, genome-wide analysis of epigenetic marks, and cell fractionation of human islets into their constitutive cell types have greatly increased our understanding of the complex processes that govern endocrine cell function in health and disease. Further progress in this area holds great promise for delivering new targets for the development of novel diabetes therapies.
\end{abstract}

Keywords Endocrine pancreas $\cdot$ Epigenetics $\cdot$ Epigenomics · Reprogramming $\cdot$ Review $\cdot$ Transcription

$\begin{array}{ll}\text { Abbreviations } \\ \text { ARNT } & \text { Aryl hydrocarbon receptor nuclear translocator } \\ \text { ChIP-Seq } & \begin{array}{l}\text { Chromatin immunoprecipitation followed by } \\ \text { high-throughput sequencing }\end{array} \\ \text { qRT-PCR } & \text { Real-time quantitative RT-PCR } \\ \text { RNA-Seq } & \text { RNA sequencing-based technology }\end{array}$

\section{The islets of Langerhans: critical regulators of blood} glucose homeostasis

Blood glucose homeostasis is achieved by the interplay between the endocrine pancreas and peripheral tissues (liver,

N. C. Bramswig $\cdot$ K. H. Kaestner $(\bowtie)$

Department of Genetics, Perelman School of Medicine,

University of Pennsylvania, 12-126 Translational Research Center,

3400 Civic Center Boulevard, Philadelphia, PA 19104, USA

e-mail: kaestner@mail.med.upenn.edu

N. C. Bramswig $\cdot$ K. H. Kaestner

Institute for Diabetes, Obesity and Metabolism, Perelman School of Medicine, University of Pennsylvania, Philadelphia, PA, USA muscle and adipose tissue). The islets of Langerhans, which form the endocrine compartment of the pancreas, are composed of five different hormone-producing cell types, of which the glucagon-secreting alpha cells and the insulinsecreting beta cells are the most important for acute regulation of blood sugar levels. Insulin secretion leads to decreased gluconeogenesis in the liver, increased glucose uptake in skeletal muscle and adipose tissue, and lowering of blood glucose levels, while the secretion of glucagon elevates blood glucose levels by enhancing hepatic gluconeogenesis and glycogen breakdown. In type 2 diabetes mellitus, insulin resistance of peripheral tissues and beta cell dysfunction cause relative hypoinsulinaemia, relative hyperglucagonaemia, and overt hyperglycaemia. The consequences of chronically high blood glucose levels are coronary heart disease, stroke, blindness and renal failure, diseases that constitute a major public health problem and a significant burden on the economy of industrialised nations, and increasingly the worldwide economy.

Environmental and genetic influences are the major contributors to type 2 diabetes, causing insulin resistance in peripheral organs and inappropriately low insulin and high glucagon secretion by pancreatic islets. Large-scale genetic mapping of patients with type 2 diabetes and healthy controls over the past 15 years, in genome-wide association studies (GWAS), had promised to elucidate the genetic causes of diabetes and lead to new treatment paradigms. However, while to date more than 60 risk loci, encompassing up to 500 genes, have been identified as contributing to the disease, even when combined these loci only explain $5-10 \%$ of diabetes risk $[1,2]$. This so-called 'missing heritability' has been suggested to be caused by the existence of many more risk variants with even smaller effect sizes than those of the risk loci identified thus far, or by combinatorial effects among multiple loci as yet to be discovered. Here, we will consider the additional possibility that environmental influences [3-6], which can be reflected by epigenetic modifications, might play a much bigger role in disease pathogenesis than previously appreciated. 
Environmental influences, such as sedentary lifestyle and unhealthy diet in adulthood, as well as unfavourably high or low blood glucose levels during fetal development, influence the risk of developing type 2 diabetes. Intrauterine malnutrition, for example during the Dutch hunger winter between 1944 and 1945, followed by a normalisation of food supply, increases the risk of the metabolic syndrome in the offspring decades later [7]. In addition, offspring of mothers with gestational diabetes mellitus, exposed to high glucose levels during development, also show an increased risk for metabolic disease phenotypes [8]. Hence, any dramatic change in blood glucose levels during fetal development has the potential to impact on metabolic homeostasis in adulthood. How is this 'metabolic memory' stored, and how can it affect the metabolism and pathogenesis of type 2 diabetes many decades after the exposure to an unfavourable environment? Epigenetic modifications, as defined below, are thought to play an important role linking environmental influences and diabetes risk.

Epigenetics is the study of phenotypic or gene expression changes caused by mechanisms other than alterations of the DNA sequence itself. Epigenetic effects can be mediated by relatively long-term molecular changes that include methylation of the cytosine base of DNA (5-mC), typically in a CpG dinucleotide context, and several classes of histone modification, such as methylation and acetylation. While high levels of $5-\mathrm{mC}$ at gene promoters generally lead to transcriptional repression, the function of histone marks depends on the type and exact location of the modification. For example, H3K4me3, the tri-methylation of lysine 4 (K4) on the core histone protein $\mathrm{H} 3$, is associated with the activation of transcription, while the H3K27me3 mark (trimethylation of lysine 27 on histone H3) is linked to repression of transcription. Indepth analysis of the epigenetic landscape of human islets as an archive of metabolic memory can provide important clues about disease pathogenesis and suggest novel pathways for therapeutic interventions. Here we will focus on the epigenetic profile of human pancreatic alpha and beta cells and their potential contribution to islet dysfunction in type 2 diabetes.

\section{The transcriptome of human pancreatic islets in health and disease}

Over the past 10 years, efforts to study the global profile of steady-state levels of mRNAs, often termed 'expression profiling' or 'transcriptome analysis', have undergone a rapid technological evolution and refinement. Early analyses using whole islet RNA samples - with the associated issues of varying islet purity and composition-have been superseded by transcriptomes of highly purified endocrine cell populations. Likewise, the analysis platforms have shifted from hybridisation of labelled cDNA fractions to probes assembled on microarrays to RNA sequencing- based technologies (RNA-Seq), which allow for much higher sensitivity and the easy detection of splice variants [9].

We begin our review with a study from 2005, when Gunton and colleagues published expression microarray and real-time quantitative RT-PCR (qRT-PCR) analyses on islets isolated from seven non-diabetic and five type 2 diabetic donors [10]. Approximately 44,000 genes were analysed on an oligonucleotide array, of which 243 genes were upregulated and 137 genes were downregulated in type 2 diabetic human islets. This surprisingly low number of differentially expressed genes detected is likely to be the result of the aforementioned technical issues stemming from the use of un-fractionated islets as the source of the RNA samples. Among the genes with significantly lower expression in diabetic samples were the MODY gene HNF4A (encoding hepatic nuclear factor $4 \alpha$ ), several genes encoding enzymes involved in glucose metabolism, and the insulin signalling mediators IRS2 and $A K T 2$. Surprisingly, many genes encoding transcription factors known to be important for islet function, such as MAFA, NKX6-1 and PDX1, were not differentially expressed. The most significantly decreased gene was $A R N T$ (encoding aryl hydrocarbon receptor nuclear translocator [ARNT]). The authors confirmed a biological role for ARNT in beta cells by analysing mice with a conditional mutation in the gene encoding this transcription factor. However-as is true for all case-control studies - it is impossible to determine to what degree the altered expression of ARNT in human islets contributes to diabetes. More recently, human islet samples were analysed using qRT-PCR and shown to exhibit a significant decrease in MAFA, MAFB, PDX1 and NKX6-1 mRNA levels [11]. The discrepancy between the two studies could be due to different purities of the islet preparations, the larger sample size used in the second study ( $n=7$ non-diabetic, $n=5$ diabetic vs $n=8$ non-diabetic, $n=8$ diabetic), or the use of islets from donors more severely affected by type 2 diabetes. In 2012, Eizirik and colleagues performed RNA-Seq analysis, which is more sensitive than array-based expression profiling [9], on whole human islets cultured under control conditions or cytokine exposure [12], focusing on the transcriptional changes following inflammatory challenge as early events in type 1 diabetes.

However, as mentioned above, the use of whole islets for the transcriptional analysis of non-diabetic and type 2 diabetic islets is problematic. First, human islets are a mixture of different cell types and consist on average of $35 \%$ alpha cells and $54 \%$ beta cells [13]. Therefore, whole islet analysis cannot determine in which cell type the mRNA changes occur, and might not detect important cell-type specific changes if they occur in the opposite direction in alpha and beta cells, for instance. Second, type 2 diabetic islets contain a lower percentage of beta cells than non-diabetic islets [14]. As a result, a decrease in expression of beta cell specific genes or an 
increase in alpha cell specific genes might simply be the result of the decreased percentage of beta cells in diabetic islets. It should be noted that any disruption of islet architecture into single cell suspensions, or the sorting event itself, might modify gene expression. In addition, the altered architecture of diabetic islets might affect their isolation, thus representing only a subpopulation of diabetic islets. Nevertheless, sorting of human islets into alpha and beta cells does allow for celltype specific analysis, which has led to novel insights into islet function.

In 2008, Dorrell and colleagues published a novel FACS strategy using a newly devised panel of cell-surface antibodies, allowing for the efficient fractionation of human pancreatic cell types and enabling cell-type specific analyses [15]. The same group used this strategy for the first genome-wide transcriptional analysis of non-diabetic endocrine and exocrine cell types [16]. This microarray-based study revealed multiple gene clusters with cell-type specific expression, and identified numerous genes differentially expressed between alpha and beta cells. Interestingly, the transcription factors aristaless related homeobox (ARX) and pancreatic and duodenal homeobox 1 (PDX1) were confirmed to be alpha and beta cell specific, respectively, while $M A F B$ was found to be expressed in both alpha and beta cells in humans, which is very different from the situation in mice, where the orthologous gene $M a f B$ is expressed specifically in alpha cells in adulthood [17]. This finding also illustrates that, while many features of endocrine cells are conserved between mouse and human, there are speciesspecific events, necessitating the analysis of human source tissue.

Subsequently, a similar sorting strategy was applied for the genome-wide transcriptional and epigenomic analysis of nondiabetic alpha, beta and exocrine cells [18]. RNA-Seq analysis revealed many genes with distinct cell-type specific expression patterns, including genes associated with type 2 diabetes risk variants, such as PTPRD among the alpha cell specific genes, and the zinc transporter SLC30A8 and potassium channels KCNQ2 and KCNJ11 among the beta cell specific genes. The establishment of a reliable sorting strategy and the cell-type specific analysis of non-diabetic islet cell types is an important prerequisite that will enable improved analyses of epigenome and transcriptome changes that occur in alpha and beta cells in type 2 diabetes.

\section{The epigenome of human islets: insights into endocrine cell plasticity}

The analysis of epigenetic modifications in human islets can elucidate their contribution to gene regulation and might help to explain how environmental influences are translated into metabolic memory to contribute to type 2 diabetes pathogenesis. As mentioned above, there are multiple different epigenetic modifications influencing gene activity, including methylation of cytosine $(5-\mathrm{mC})$ and multiple enzymatic modification of the histone proteins that make up the nucleosome and control chromatin structure.

In 2010, chromatin from human islets was profiled using formaldehyde-assisted isolation of regulatory elements followed by high-throughput sequencing (FAIRE-Seq) analysis, a technology that allows for the determination of open chromatin regions, which are enriched for active regulatory elements [19]. Gaulton and colleagues identified 340 genes with isletselective open chromatin regions near the transcriptional start site or in the gene body, among them $P D X 1, N K X 6-1$ and $S L C 30 A 8$, as expected, and noticed that regions of open chromatin were often organised into clusters. They identified 3,348 islet-selective COREs (clusters of open chromatin regulatory elements), including COREs at the PDX1 and TCF7L2 loci. Interestingly, analysis of risk alleles showed that one of the TCF7L2 variants (rs7903146) was present in a FAIREenriched site. Importantly, the chromatin state at this site was more open in chromosomes carrying the type 2 diabetes risk allele (T), consistent with previous reports of increased transcription of the TCF7L2 gene in islets with this T allele [20].

Chromatin immunoprecipitation followed by highthroughput sequencing analysis (ChIP-Seq) can be used for genome-wide transcription factor binding and histone modification profiling [21]. This technique was performed for the profiling of different histone modifications, such as H3K4me3, H3K27me3, H3K4me1 and H3K79me2, as well as CCCTC factor (CTCF) binding sites in whole human islets $[22,23]$. It became apparent that cell-type specific analyses of the histone modification landscapes were needed to draw conclusions about the epigenetic regulation of alpha and beta cells. ChIP-Seq analysis of sorted human alpha and beta cells revealed that these cell types exhibit similar monovalent $\mathrm{H} 3 \mathrm{~K} 4 \mathrm{me} 3$ and $\mathrm{H} 3 \mathrm{~K} 27 \mathrm{me} 3$ profiles, but that alpha cells show a much higher number of bivalently marked genes than either exocrine cells or beta cells [18]. Remarkably, many developmental regulators and beta cell specific transcription factors were among the bivalently marked genes in alpha cells, providing a likely explanation for the previously described transdifferentiation potential of mammalian alpha cells [24-26], and suggesting alpha cell specific epigenomic plasticity. Intriguingly, treatment of human islets with a histone methyltransferase inhibitor caused a partial conversion of alpha cells towards the beta cell fate, indicating that epigenomic modulators might serve as a means to reprogramme endocrine cells to develop as beta cells [18].

Thus far, only a few studies have analysed DNA methylation levels in human pancreatic islets, focusing on the analysis of selected loci. For instance, methylation levels of the enhancer and distal promoter region of $P D X 1$ have been shown 
to be increased in type 2 diabetic human islets, possibly contributing to the decreased $P D X 1$ mRNA levels observed in such tissue [27]. Volkmar and colleagues have analysed human islet DNA methylation profiles from five type 2 diabetic and 11 non-diabetic cadaveric organ donors identifying 276 differentially methylated $\mathrm{CpG}$ sites [28]. To our knowledge, genome-wide DNA methylation levels of human alpha and beta cells have yet to be determined. It would be highly interesting to combine genome-wide cell-type specific histone modification and DNA methylation profiles for the assembly of epigenomic roadmaps of human alpha and beta cells to fully elucidate the epigenome of these diabetes-relevant cell types.

In summary, cell-type specific histone modification and transcriptional analyses have uncovered important differences between human alpha and beta cells, and have suggested that targeted epigenetic manipulation might promote alpha to beta cell fate conversion. In the future, these studies need to be extended to the analysis of non-diabetic and type 2 diabetic alpha and beta cells to assess cell-type and disease-state specific transcriptional and epigenomic differences, to increase our understanding of the pathogenesis of type 2 diabetes, and to elucidate a possible role of epigenetic regulation of diseasestate specific genes.

Funding Related work in the Kaestner lab is funded through NIH grants R01 DK088383 and U01 DK089529.

Duality of interest The authors declare that there is no duality of interest associated with this manuscript.

Contribution statement Both authors were responsible for the conception and design of the manuscript, drafting the article and revising it critically for important intellectual content. Both authors approved the version to be published.

\section{References}

1. Voight BF, Scott LJ, Steinthorsdottir V et al (2010) Twelve type 2 diabetes susceptibility loci identified through large-scale association analysis. Nat Genet 42:579-589

2. Morris AP, Voight BF, Teslovich TM et al (2012) Large-scale association analysis provides insights into the genetic architecture and pathophysiology of type 2 diabetes. Nat Genet 44:981-990

3. Hu FB (2011) Globalization of diabetes: the role of diet, lifestyle, and genes. Diabetes Care 34:1249-1257

4. Knowler WC, Barrett-Connor E, Fowler SE et al (2002) Reduction in the incidence of type 2 diabetes with lifestyle intervention or metformin. N Engl J Med 346:393-403

5. van Dam RM (2003) The epidemiology of lifestyle and risk for type 2 diabetes. Eur J Epidemiol 18:1115-1125

6. Yach D, Stuckler D, Brownell KD (2006) Epidemiologic and economic consequences of the global epidemics of obesity and diabetes. Nat Med 12:62-66
7. Hales CN, Barker DJ (2001) The thrifty phenotype hypothesis. Br Med Bull 60:5-20

8. Lehnen H, Zechner U, Haaf T (2013) Epigenetics of gestational diabetes mellitus and offspring health: the time for action is in early stages of life. Mol Hum Reprod 19:415-422

9. Wang Z, Gerstein M, Snyder M (2009) RNA-Seq: a revolutionary tool for transcriptomics. Nat Rev Genet 10:57-63

10. Gunton JE, Kulkarni RN, Yim S et al (2005) Loss of ARNT/HIF1beta mediates altered gene expression and pancreaticislet dysfunction in human type 2 diabetes. Cell 122:337-349

11. Guo S, Dai C, Guo M et al (2013) Inactivation of specific beta cell transcription factors in type 2 diabetes. J Clin Invest 123: 3305-3316

12. Eizirik DL, Sammeth M, Bouckenooghe T et al (2012) The human pancreatic islet transcriptome: expression of candidate genes for type 1 diabetes and the impact of pro-inflammatory cytokines. PLoS Genet 8:e1002552

13. Brissova M, Fowler MJ, Nicholson WE et al (2005) Assessment of human pancreatic islet architecture and composition by laser scanning confocal microscopy. J Histochem Cytochem 53:1087-1097

14. Weir GC, Bonner-Weir S (2013) Islet beta cell mass in diabetes and how it relates to function, birth, and death. Ann N Y Acad Sci 1281: 92-105

15. Dorrell C, Abraham SL, Lanxon-Cookson KM, Canaday PS, Streeter PR, Grompe M (2008) Isolation of major pancreatic cell types and long-term culture-initiating cells using novel human surface markers. Stem Cell Res 1:183-194

16. Dorrell C, Schug J, Lin CF et al (2011) Transcriptomes of the major human pancreatic cell types. Diabetologia 54:2832-2844

17. Artner I, Le Lay J, Hang Y et al (2006) MafB: an activator of the glucagon gene expressed in developing islet alpha- and beta-cells. Diabetes 55:297-304

18. Bramswig NC, Everett LJ, Schug J et al (2013) Epigenomic plasticity enables human pancreatic alpha to beta cell reprogramming. J Clin Invest 123:1275-1284

19. Gaulton KJ, Nammo T, Pasquali L et al (2010) A map of open chromatin in human pancreatic islets. Nat Genet 42:255-259

20. Helgason A, Palsson S, Thorleifsson G et al (2007) Refining the impact of TCF7L2 gene variants on type 2 diabetes and adaptive evolution. Nat Genet 39:218-225

21. Park PJ (2009) ChIP-seq: advantages and challenges of a maturing technology. Nat Rev Genet 10:669-680

22. Bhandare R, Schug J, Le Lay J et al (2010) Genome-wide analysis of histone modifications in human pancreatic islets. Genome Res 20: 428-433

23. Stitzel ML, Sethupathy P, Pearson DS et al (2010) Global epigenomic analysis of primary human pancreatic islets provides insights into type 2 diabetes susceptibility loci. Cell Metab 12:443-455

24. Yang YP, Thorel F, Boyer DF, Herrera PL, Wright CV (2011) Context-specific alpha- to-beta-cell reprogramming by forced Pdx1 expression. Genes Dev 25:1680-1685

25. Thorel F, Nepote V, Avril I et al (2010) Conversion of adult pancreatic alpha-cells to beta-cells after extreme beta-cell loss. Nature 464: $1149-1154$

26. Collombat P, Xu X, Ravassard P et al (2009) The ectopic expression of Pax4 in the mouse pancreas converts progenitor cells into alpha and subsequently beta cells. Cell 138:449-462

27. Yang BT, Dayeh TA, Volkov PA et al (2012) Increased DNA methylation and decreased expression of PDX-1 in pancreatic islets from patients with type 2 diabetes. Mol Endocrinol 26: $1203-1212$

28. Volkmar M, Dedeurwaerder S, Cunha DA et al (2012) DNA methylation profiling identifies epigenetic dysregulation in pancreatic islets from type 2 diabetic patients. EMBO J 31:1405-1426 